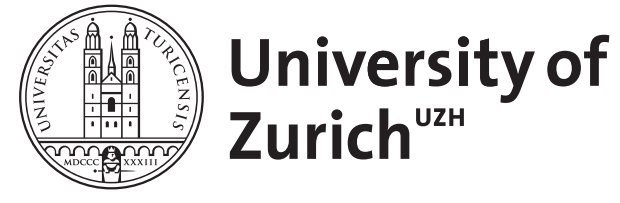

\title{
La guerre et les maladies vénériennes
}

\author{
Bloch, Bruno
}

\begin{abstract}
Parmi toutes les misères matérielles que la guerre mondiale a déchaînées sur l'humanité, les ravages causés par le fléau vénérien occupent une place considérable. En vérité nous ne somraes pas encore, aujourd'hui, en état d'entrevoir les multiples rapports qui relient les maladies vénériennes à la guerre; il nous manque des données statistiques exactes portant sur le nombre total des soldats vénériens dans les différentes armées, et plus incomplètes encore sont nos connaissances sur l'extension des maladies vénériennes dans la population civile des pays belligérants. Incomplets aussi sont nos renseignements sur toutes les mesures prises par les différents pays et armees pour l'endiguement des maladies vénériennes, et surtout sur leurs résultats favorables et défavorables. Malgré ces lacunes, les faits acquis par l'expérience sont si nombreux ct importants qu'ils doivent attirer l'attention de tout homme à qui l'avenir de l'humanité civilisée tient à cœur. La guerre mondiale nous a montré que la lutte contre les maladies vénériennes est un problème qui nécessite d'urgence une prompte solution. Elle a aussi modifié dans leur essence nos opinions sur les moyens les mieux appropriés à cette solution. Il y a là, à côté de tous les maux et de toutes les tristesses que la guerre a semés, un bon grain à la germination duquel chaque peuple et chaque race sont intéressés
\end{abstract}

DOI: https://doi.org/10.1017/s1026881200033420

Posted at the Zurich Open Repository and Archive, University of Zurich

ZORA URL: https://doi.org/10.5167/uzh-154547

Journal Article

Published Version

Originally published at:

Bloch, Bruno (1920). La guerre et les maladies vénériennes. International Review of the Red Cross, $2(15): 259-275$.

DOI: https://doi.org/10.1017/s1026881200033420 


\section{PROF.-Dr BRUNO BLOCH,}

Directeur de la Clinique dermatologique de l'Université de Zurich.

\section{La guerre et les maladies vénériennes.}

Parmi toutes les misères matérielles que la guerre mondiale a déchaînées sur l'humanité, les ravages causés par le fléau vénérien occupent une place considérable. En vérité nous ne sommes pas encore, aujourd'hui, en état d'entrevoir les multiples rapports qui relient les maladies vénériennes à la guerre; il nous manque des données statistiques exactes portant sur le nombre total des soldats vénériens dans les différentes armées, et plus incomplètes encore sont nos connaissances sur l'extension des maladies vénériennes dans la population civile des pays belligérants. Incomplets aussi sont nos renseignements sur toutes les mesures prises par les différents pays et armées pour l'endiguement des maladies vénériennes, et surtout sur leurs résultats favorables et défavorables. Malgré ces lacunes, les faits acquis par l'expérience sont si nombreux et importants qu'ils doivent attirer l'attention de tout homme à qui l'avenir de l'humanité civilisée tient à cœur. La guerre mondiale nous a montré que la lutte contre les maladies vénériennes est un problème qui nécessite d'urgence une prompte solution. Elle a aussi modifié dans leur essence nos opinions sur les moyens les mieux appropriés à cette solution. Il y a là, à côté de tous les maux et de toutes les tristesses que la guerre a semés, un bon grain à la germination duquel chaque peuple et chaque race sont intéressés.

Avant la guerre déjà, dans bien des milieux, on se rendait parfaitement compte que la lutte contre le fléau vénérien était un des principaux devoirs de l'hygiène sociale et de la conservation de la race. On savait que ces maladies sont parmi les plus répandues et qu'il n'y a pas d'âge, de condition, de race et de climat qu'elles épargnent. L'expérience médicale leur découvre chaque jour de nouvelles suites nuisibles et irréparables. Celles-ci ne s'étendent pas seulement à l'individu malade - dont elles touchent souvent, hélas, les organes 


\section{La guerre et les maladies vénériennes.}

vitaux - mais aussi au plus proche entourage et jusqu'a la seconde génération. Elles détruisent finalement le bonheur de la famille et sapent ainsi à la base le développement du peuple et de l'Etat. C'est ainsi qu'on estime, pour ne citer que quelques exemples, le déficit annuel des naissances dû, en Allemagne, à la stérilité gonorrhoïque à environ 200,000. Parmi les enfants aveugles, à Londres, le $2 \%$ sûrement, le $2,8 \%$ très probablement, doivent leur infirmité à la syphilis de leurs parents ; de même pour le $7 \frac{1}{2} \%$ des sourds. Dans les asiles d'aveugles en Allemagne, le nombre de ceux qui le sont par suite de blennorrhagie ne s'élève pas à moins de $25 \%$.

On s'est donné beaucoup de peine ces dernières années pour arriver à connaître le nombre des personnes atteintes de maladies vénériennes. Cependant aucune des nombreuses statistiques qui ont été faites à ce sujet ne résiste à une sévère critique. Les recherches les plus exactes établissent que dans les grandes villes un homme sur 4 ou 5 a la syphilis. Beaucoup plus répandue est cette autre maladie vénérienne, la blennorrhagie. C'est un fait prouvé que dans les grandes villes, presque chaque homme en a été une fois atteint, beaucoup même deux ou trois fois et même plus.

Les enquêtes statistiques ont encore fourni sur l'extension et l'origine des maladies vénériennes d'autres très précieux éclaircissements. Ces maladies sont d'autant plus nombreuses que les hommes vivent plus à l'étroit, et ont leur fréquence minimale à la campagne, maximale dans les grandes villes. C'est ainsi qu'au Danemark le pourcentage des maladies vénériennes comporte : à Copenhague $20 \%$, dans les villes de la province $3 \%$, à la campagne $0,4 \%$. En outre les maladies vénériennes sont en très grand nombre contractées dans le jeune âge, fait d'importance capitale dans la question des mesures préventives.

D'après une statistique de Fournier, le $38 \%$ des femmes atteintes de maladies vénériennes s'est contaminé entre $I_{4}$ et $2 \mathrm{I}$ ans, et d'après Brandweiner le $67 \%$ des hommes s'infecte avant 26 ans. 


\section{La guerre et les maladies vénériennes.}

De telles expériences avaient déjà conduit, il y a 20 ans, de nombreux médecins et philanthropes de tous pays réunis à $\mathrm{Bru-}$ xelles, à l'instigation du regretté $\mathrm{D}^{\mathrm{r}}$ Dubois-Havenith, à étudier la manière d'arrêter l'extension toujours croissante des maladies vénériennes. De cette réunion mémorable date la lutte organisée contre le fléau vénérien. Et de ce jour elle a été entreprise avec plus ou moins d'intensité par la plupart des pays. civilisés et poursuivie par certains avec une grande énergie. Sans nul doute elle a déjà produit de bons résultats, bien que ceux-ci ne soient pas appréciables en chiffres, mais il n'en reste pas moins que le plus gros du travail est encore à faire et que le combat doit être mené avec de tout autres moyens et infiniment plus d'énergie que par le passé, si le but veut être atteint dans un avenir plus ou moins rapproché.

Cela, la guerre vient de nous le démontrer avec une saisissante netteté, C'est un fait d'histoire, toujours à nouveau confirmé, que les époques guerrières son tfavorables à la propagation des maladies vénériennes. Déjà la première grosse épidémie de syphilis qui sévit sur l'Europe se rattachait à un épisode guerrier : l'occupation de Naples par Charles d'Anjou, en r493. Cette constatation historique est en soi bien facile à expliquer. L'éloignement du foyer et de la famille, le transfert dans un milieu absolument étranger, le contact d'éléments. nouveaux, souvent rien moins que bons, l'entassement de grosses masses d'hommes jeunes et souvent sans scrupules, l'excitation et la séduction exercées par de mauvaises femmes, sont tout autant de facteurs qui en temps de paix déjà, par exemple lors de la transplantation des habitants des campagnes dans les grandes villes, agissent dans un sens défavorable, et qui en temps de guerre ont un effet plus nuisible encore. A cela s'ajoute pour l'homme d'une part l'incertitude du sort qui fait perdre toute prudence et toute retenue contre sa contamination et celle des autres, et qui le pousse à ne pas laisser échapper l'éphémère jouissance $\mathrm{du}$ moment, et d'autre part la domination souveraine en territoire envahi, qui fait perdre bien vite à beaucoup d'hommes tout sens moral. Il n'y a par suite-

$$
-26 \mathrm{I}-
$$




\section{La guerre et les maladies vénériennes.}

rien d'étonnant que dans cette guerre aussi, la plus grande de toutes, l'extension des maladies vénériennes ait pris une pareille ampleur. Cela ne tient pas tant au fait que le pourcentage des vénériens était particulièrement grand, mais bien plus à cette circonstance, que dans aucune autre précédente guerre encore d'aussi grandes masses d'hommes n'ont été arrachées à leur ambiance habituelle et jetées sur les champs de bataille. D'autres circonstances ont encore exercé une grande influence : en temps de paix les maladies vénériennes, ainsi que la remarque en a déjà été faite, étaient chez nous surtout le résultat de la vie citadine; le paysan jusqu'à ces derniers temps, a échappé, si non tout à fait, du moins en majeure partie à ce fléau. Là aussi la guerre vient d'apporter une profonde modification, dont les suites sont encore incalculables. Une grosse partic de la population campagnarde, et justement la plus robuste et la plus laborieuse, a eu, des années durant, l'occasion de s'infecter. D'où le danger que beaucoup de ces paysans aient rapporté au pays leur maladie et qu'ils y deviennent le point de départ de nouveaux foyers d'infection, justement dans des contrées où jusque-là ces maladies, à l'encontre de la tuberculose, ne présentaient pas grand danger. Cette éventualité de la contamination de la population campagnarde de l'Europe m'apparaît comme un des côtés les plus sombres du problème. Elle prend une importance toute spéciale dans les pays où la démobilisation ne s'est pas effectuée en bon ordre.

Les maladies vénériennes n'ont pas seulement causé des victimes au front parmi les troupes, mais aussi à l'arrière, où la population civile révèle une augmentation considérable de la morbidité. Cette augmentation touche la population masculine adulte et s'explique par les hauts salaires de gnerre et le relâchement des mœurs; malheureusement elle s'étend aussi aux femmes et à la jeunesse. C'est ainsi qu'à Vienne par exemple, on a constaté qu'alors que le nombre des vénériens de sexe masculin âgés de 15 à I 8 ans était en r 913 de $4 \%$ il s'est élevé en $19 I_{5}$ à II \% c'est dans une proportion semblable que s'est 


\section{La guerre et les maladies vénériennes.}

accru le pourcentage des femmes mariées atteintes de maladies vénériennes. A l'armée aussi on a été frappé du très grand nombre d'hommes mariés parmi les vénériens.

Il serait à présent très important et intéressant de savoir le chiffre absolu et proportionnel des gonorrhoïques et syphilitiques dans les différentes armées; ces données nous permettraient d'estimer d'un coup d'œil la grandeur du désastre. Malheureusement une telle estimation, est aujourd'hui encore impossible. Je dois seulement me contenter ici de donner quelques exemples:

En Allemagne, pendant la guerre de $1870-7$ I déjà, le nombre des soldats vénériens était monté de $16 \%$ à $90 \%$, de sorte que sur 800,000 hommes il n'y avait pas moins de 34,000 malades. Pour la dernière guerre les chiffres ne sont pas encore exactement connus; d'après Linser il y aurait eu dans l'armée I $5 \%$ nouveaux infectés par an; bien pires seraient les proportions dans les troupes d'étapes et dans l'armée territoriale (environ $29 \% 0$ ).

En Autriche, en temps de paix déjà, les maladies vénériennes représentaient le I7 au $3 \mathrm{I} \%$ de tous les cas de maladies, et nécessitaient par an 638,139 jours d'infirmerie. Le nombre des lits $(\mathrm{r} 2,577)$ dont les trois commandements militaires de Vienne, Budapest et Agram disposaient pour leurs vénériens, nous montre dans quelle mesure la guerre a accru ces chiffres. Cela représente, en comptant la durée moyenne de maladie a 35 jours, environ 125,000 malades par an.

D'un rapport du "Inter-Departmental Committee on infections diseases " j'extrais les intéressantes données suivantes:

Dans l'armée canadienne le nombre annuel des soldats atteints de maladies vénériennes était en r $915: 222 \%$ (soit un vénérien sur 5 hommes) ; en I9I6:209\% ; en I9I $7:$ II $4 \%$; en IgI $8: 8 \mathrm{I} \%$. Dans l'armée australienne le pourcentage variait entre II $8 \%$ et $184 \%$.

Dans l'armée britannique le nombre annuel des vénériens oscillait, avant la guerre, entre 30 et $50 \%$. Les chiffres exacts de la dernière guerre nous manquent encore, mais il est admis

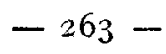




\section{La guerre et les maladies vénériennes.}

que la morbidité était, dans l'armée britannique, sensiblement moindre que dans les troupes canadiennes et australiennes.

Dans l'armée américaine, ces chiffres sont: pour I9I5, $83 \%$; I9I6, 90\% ; 1917 et 18 , d'après Emerson, $52 \%$ et $75 \%$; depuis septembre r 9 r $8,30 \%$ à $56,8 \%$.

En Suisse, pour ne citer qu'un pays resté neutre, l'établissement sanitaire à Soleure a soigné, de août IgI4 à janvier IgI 8 , 3709 soldats vénériens, avec un chiffre total de 183,021 jours d'infirmerie. Cela représente, en comptant les jours de convalescence, le service de 3,000 hommes pendant $7^{6}$ jours.

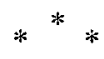

Ces quelques données doivent suffire pour montrer clairement quelle énorme importance ont pris les maladies vénériennes pendant la guerre mondiale. Si l'on représente sa longue durée, les millions d'hommes qui y ont pris part, si l'on songe en outre que les conditions dans la plupart des armées de l'Est de l'Europe étaient encore bien pires et que chaque soldat non guéri, rentré dans son foyer, y devient un centre nouveau d'extension pour la syphilis et la gonorrhée, on est bien obligé d'avouer que de tous les problèmes d'hygiène sociale que la guerre nous a laissés après elle, celui de la lutte contre les maladies vénériennes est bien le plus urgent et le plus important.

De nombreux médecins dans les pays belligérants, ainsi que les gouvernements et les chefs d'armées, se sont bien vite rendus à cette évidence. Déjà en 1914 , peu de mois après la déclaration de la guerre, surgissaient de partout des voix qui réclamaient des mesures énergiques contre l'énorme extension des maladies vénériennes à l'armée et dans le pays. Sans quoi il en résulterait inévitablement un notable amoindrissement de la puissance combattive des troupes et un dommage durable pour le peuple. Ces voix ne restèrent pas sans réponse. Bientôt on vit paraitre dans tous les pays qui se trouvaient en guerre, des rapports sur les mesures à prendre en vue de la lutte contre les maladies vénériennes sur le front et à l'intérieur. 


\section{La guerre et les maladies vénériennes.}

Ces rapports nous apprennent : tout d'abord, qu'aucun pays ne se trouvait suffisamment préparé pour ce devoir. Partout, à l'exception peut-être de l'Amérique, qui entra plus tard en guerre, la réglementation première fut de tous points improvisée et ce ne fut que peu à peu, à la suite de l'expérience acquise, qu'elle fit place à une organisation vaste et méthodique.

Le but de toutes ces institutions fut naturellement, aussi longtemps que dura la guerre, de diminuer autant que possible les pertes des soldats rendus inaptes par les maladies vénériennes, en d'autres termes le maintien de la force combattive de l'armée, et ce n'est qu'en seconde ligne que figurèrent la considération de la population civile et la préoccupation de l'après-guerre.

Les systèmes qui ont été adoptés par les différents pays et armées pour la lutte contre les maladies vénériennes se différencient fortement les uns des autres, selon la mentalité du peuple et de la troupe, selon l'esprit qui dominait en haut lieu, enfin selon les moyens disponibles. Plus d'une fois l'insuffisance des résultats obtenus fit changer l'organisation. Ce fut le cas, par exemple, pour l'armée d'expédition américaine qui, pour commencer, adopta le système français, mais introduisit, au bout d'un certain temps une organisation particulière et toute différente. Naturellement il est impossible de discuter ici les différents systèmes et leurs résultats. Pour cela ne suffisent ni la place dont je dispose, ni les matériaux que les organes officiels ont réunis jusqu'à aujourd'hui. Les publications faites jusqu'à ce jour nous montrent que presque tout a été essayé, depuis le simple appel à la morale et au sentiment de responsabilité de l'individu pris à part, depuis l'encouragement à l'abstinence sexuelle totale, jusqu'aux mesures de discipline militaire les plus draconiennes. Lorsque tous les résultats et tous les échecs seront connus, alors nous serons en possession de documents qui auront, pour la continuation de la campagne contre les dangers sexuels une inestimable valeur.

En général, aux cours de mes recherches dans ce domaine, 


\section{La guerre et les maladies vénériennes.}

j'ai eu l'impression nette que les résultats obtenus étaient toujours meilleurs par l'introduction de mesures de contrainte et de menaces de peine, plutôt que par l'appel au sens moral et au bon vouloir de chaque individu. En temps de guerre les conditions sont à ce point de vue particulières et différentes de celles du temps de paix; elles permettent beaucoup plus facilement, les droits de l'individu se trouvant réduits à un minimum, l'application de mesures de contrainte et de peines disciplinaires. D'un rapport de la commission anglaise d'Etat ressort encore le fait important que le succès de mesures prophylactiques dans un corps de troupes, dépend dans un très haut degré du zèle et du dévouement que les médecins responsables mettent à l'accomplissement de leurs devoirs. Sans aucun doute ce facteur joue en temps de paix aussi un rôle important.

En principe les devoirs qui incombent aux services sanitaires dans la lutte contre les maladies vénériennes sont en temps de guerre les mêmes qu'en temps de paix. Seuls les moyens diffèrent en partie. Ces devoirs peuvent être précisés dans les points suivants :

$I^{\circ}$. Tarissement des sources par la diminution, l'assainissement et la surveillance sanitaire du rapport sexuel illégitime, avant tout de la prostitution.

$2^{0}$. Appel adressé au côté mâle : encouragement à l'abstinence ou du moins invitation à une grande modération de la vie sexuelle eu égard aux inévitables dangers moraux et physiques qui l'accompagnent - bref, instruction, éducation sexuelles et éventuellement peines disciplinaires appropriées en cas d'infraction aux prescriptions. Avertissement sur les dangers de l'alcoolisme.

$3^{\circ}$. Prophylaxie personnelle. Elle consiste ou bien en ce que chaque soldat soit pourvu d'un "nécessaire prophylactique " employé avant et après l'acte sexuel, ou bien dans l'installation des stations prophylactiques où le soldat doit se rendre aussitôt que possible après l'acte. Ces deux procédés manquent totalement leur but si leur exécution est livrée au bon 


\section{La guerre et les maladies vẻnériennes..}

vouloir de chacun. Pour qu'ils soient couronnés de succès il faut en rendre l'application obligatoire. Le soldat qui acquiert une maladie vénérienne et qui ne peut pas prouver qu'il s'est soumis aux mesures prophylactiques, sera puni. Cela nécessite d'un autre côté l'instruction approfondie et répétée de la troupe et l'instruction d'un contrôle sanitaire serré et régulier.

$4^{\circ}$. Les soldats atteints de blennorrhagie et de syphilis sont à éloigner immédiatement du service et à guérir aussi vite que possible dans les lazarets dirigés par des spécialistes. Le diagnostique de la maladie et son traitement doivent être confiés à des spécialistes expérimentés.

Toutes ces mesures ont été introduites de façon systématique dans l'armée américaine et, d'après le rapport d'Emerson à la " $4^{\text {me }}$ session plénière de la commission sanitaire des pays alliés» (mars 1919), suivies de remarquables résultats. C'est ainsi que dans le secteur de Bordeaux la morbidité est tombée de $200 \%$ à $80 \%$. - De semblables essais, nécessairement modifiés selon les lieux et les circonstances, ont été souvent appliqués dans d'autres armées, telle que l'armée allemande, avec de bons résultats.

Tout ce que nous venons de citer jusqu'à présent est, ainsi. que la remarque en a déjà été faite, sorti en premier lieu des cercles militaires dirigeants, avec comme but primordial, l'amoindrissement de l'influence néfaste des maladies vénériennes sur l'état et la force combative de l'armée. Les expériences acquises au front et aux étapes ont cependant partout exercé une action durable sur les autorités civiles et sur la population restée au foyer. On a bien vite dû reconnaître, et cela aussi dans les cercles qui auparavant se tenaient les plus éloignés de cette question, qu'il y a là un problème dont la portée n'intéresse pas seulement la jeunesse capable de porter les armes, mais l'avenir de toute la nation. Il en est résulté dans presque tous les pays et ceux même qui n'ont pas pris part à la guerre, un mouvement officiel et privé ayant pour but l'endiguement et la destruction du fléau vénérien. Ces 


\section{La guerre et les maladies vénériennes.}

mouvements sont quant à leur ampleur, leur intensité et leur organisation naturellement différents dans chaque pays, et il n'est pas possible de les suivre quelque peu en détails. Je dois bien plutôt me limiter à quelques indications.

Il existe pour la lutte contre les maladies vénériennes plusieurs méthodes différentes dans leurs principes et leurs moyens, et je voudrais tout de suite ajouter que loin de s'exclure, ces méthodes se complètent mutuellement.

Une d'elles s'adresse à la raison et au bon vouloir de l'individu; ses moyens d'action sont l'instruction et l'éducation sexuelles. Elle commence avec l'éducation de l'enfant, elle rend les jeunes gens, mûrs au point de vue sexuel, attentifs aux dangers des rapports illegitimes, elle les renseigne sur les moyens de se préserver de l'infection et leur inculque le désir, si une fois ils sont infectés, de faire tout leur possible pour se faire soigner sans tarder par des personnes compétentes, et à ne transmettre en aucun cas leur maladie.

Une autre méthode cherche à tarir si possible les maladies vénériennes dans leurs sources, qui sont la prostitution et les rapports illégitimes. Pour ce fait il ne suffit pas seulement d'une influence moralisatrice, mais d'un profond remaniement social. Car, aussi bien le mariage tardif de l'homme, que la prostitution de la femme, sont des manifestations jusqu'à aujourd'hui intimement liées à la structure de notre édifice social actuel.

Enfin on peut combattre les maladies vénériennes, par analogie avec d'autres maladies épidémiques et endémiques, au moyen des ordonnances d'Etat. Il faut alors exiger que chaque cas nouveau ou négligé de blennorrhagie et de syphilis soit dénoncé à un service de santé publique, comme on le fait pour le typhus, le choléra ou n'importe quelle autre maladie infectieuse. Le traitement par un médecin diplômé devra être poursuivi jusqu'à la guérison ou du moins jusqu'à extinction du danger d'infection. Il faudra en conséquence pourvoir a ce que l'indigent puisse se faire traiter gratis et sans interruption de son travail. Les individus malades de l'un et l'autre 


\section{La guerre et les maladies vénériennes.}

sexe qui ne donneraient pas suite à cette ordonnance et deviendraient par là un danger pour les autres, devraient être internés dans un hôpital, etc.

Il est difficile de trancher laquelle de ces méthodes offre le plus de chances de succès. Je suis pour mon compte persuadé que seule une combinaison de tous ces moyens peut conduire au but. En tout cas je ne crois pas qu'on atteigne un résultat défnitif sans application d'une réglementation spéciale d'épidémie. Je sais très bien que l'introduction d'une réglementation légale dans un domaine qui, comme celui de la vie sexuelle, est de nature si intime et si délicate et s'entoure de tant de mystères, apparaît encore aujourd'hui à la plupart comme une brutale et insupportable atteinte aux droits de la personnalité. Néanmoins je prétends que cette voie, à la longue, ne pourra pas être évitée, et j'appuie cette assertion sur les considérations suivantes. L'éducation et l'instruction, l'encouragement à la continence, la facilitation des possibilités de traitement, bref tout ce dont l'exécution suppose l'intelligence et le bon vouloir de l'individu; ne saurait écarter totalement des dangers qu'alimente l'instinct; tout cela échouera justement auprès de ceux dont on aurait le plus besoin d'être protégé, c'est-à-dire des hommes sans conscience, égoistes et légers. Et c'est là une expérience vieille comme le monde, car elle est intimement liée à l'imperfection de l'être humain. Les observations du temps de paix comme celles du temps de guerre l'ont sans cesse à nouveau confirmée, et seuls des utopistes peuvent encore espérer dans un avenir prochain un changement fondamental de la nature de l'homme.

Dans la défense de cette opinion je m'appuie entre autres sur les études poursuivies par mon assistant, le $D^{r}$ Peter, à la policlinique dermatologiquc de Zurich. Elles montrent que seule la moitié des patients atteints de maladies vénériennes suivent le conseil du médecin et se laissent régulièrement traiter. Le reste abandonne le traitement avant la guérison et propage sans scrupule la maladie, bien que tout le traitement s'effectue gratis, sans troubler en rien la vie professionnelle du malade, 


\section{La guerre et les maladies vénériennes.}

et que chaque patient ait été sérieusement mis en garde contre tous les dangers d'une maladie vénérienne non guérie. Partout où de telles recherches ont été entreprises (par exemple à Boston, Hambourg, Paris, etc.) on a constaté le même fait. Cela montre combien il faut peu compter dans de telles questions avec l'intelligence de l'individu, même dans les pays où l'instruction scolaire est générale et obligatoire. D'un côté les expériences du temps de guerre et surtout celles faites par les Américains - c'est-à-dire par un peuple dont le sentiment de liberté individuelle est très développé - nous ont montré que dans certaines conditions les droits de la liberté individuelle in sexualibus supportent très bien d'être notablement limités. Bien entendu on ne peut pas sans autre appliquer au temps de paix les conditions du temps de guerre. Mais les mœurs et coutumes dans le domaine sexuel ne sont pas constantes et immuables, elles sont, ainsi qu'un coup d'œil dans l'histoire de la civilisation le montre, soumises à de continuelles modifications selon les temps et les lieux. Qui nous dit qu'on n'arrivera pas à modifier les conceptions au point que, ce qui nous paraît être aujourd'hui une brutale et inacceptable violence, sera demain admis de bon gré et tout naturellement dans l'intérêt de la collectivité. Travailler à ce revirement me paraît être une des tâches, la plus nécessaire et atile, de la campagne antivénérienne.

On ne peut méconnaître que les tendances qui se sont fait jour depuis et par la guerre dans les différents pays, ne tendent en général à une plus ou moins grande réglementation de toute cette matière par l'Etat.

Parmi ces mesures offcielles qui ont été prises pendant les années de guerre, je signalerai en premier lieu, celles d'un Etat neutre, la Suède. Ce pays, précédé dans cette matière par les deux autres pays du Nord, a, en I9I8, mis en vigueur une loi sur la lutte contre les maladies vénériennes, qui peut servir d'exemple à bien des points de vue. Dans cette loi très moderne, se trouve réalisée une série de postulats très importants : chaque vénérien est tenu de se faire traiter médicalement et

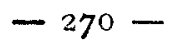




\section{La guerre et les maladies vénériennes.}

a par suite le droit au traitement gratuit. La conclusion d'un mariage est interdite avant la guérison totale. L'Etat pourvoit dans tout le pays à ce qui est nécessaire au traitement (hôpitaux, policliniques, etc.). Le médecin est tenu de renseigner chaque vénérien sur la nature et la portée de sa maladie et sur les obligations qui en découlent, de s'enquérir de la source d'infection et de la dénoncer aux autorités sanitaires. Les malades qui n'obéissent pas à ces prescriptions seront forcés de se traiter et éventuellement hospitalisés. La réglementation de la prostitution est abolie. Toute la conduite et la surveillance de la lutte contre les maladies vénériennes est entre les mains d'autorités sanitaire 3 spéciales, et la police n'a plus rien à y voir. La plus grande discrétion est assurée.

Le système suédois dont nous venons d'esquisser un court résumé réalise jusqu'à aujourd'hui, de beaucoup, la plus rationnelle et la plus progressiste des réglementations de la lutte antivénérienne par l'Etat. Les résultats et expériences que ce système nous fournira seront de toute importance pour la conduite future de la lutte. De nombreux autres pays ont produit de remarquables efforts, qui sans pouvoir être considérés comme mesures définitives et parfaites, marquent néanmoins de réels progrès.

L'Allemagne, qui avant l'introduction des dispensaires avait déjà fait beaucoup pour la lutte contre les maladies vénériennes, a élaboré en I9I8 un projet de loi qui réalise un grand progrès sur l'ancien état de choses. Il se rapproche par certains côtés de la loi suédoise (obligation de traitement, punition du rapport sexuel durant le temps de maladie, défense de contracter mariage, interdiction de tout traitement pratiqué par d'autres personnes que les médecins diplômés), sans en adopter toutefois toutes les conséquences.

En Angleterre aussi la lutte contre les maladies vénériennes a pris, sous l'influence des expériences faites au cours de la guerre et à la suite de l'intervention de l'Etat, un essor considérable. Je citerai parmi les mesures prises : la suppression des charlatans, des moyens de guérison secrets, des réclames 


\section{La guerre et les maladies vénériennes.}

publiques -- tout autant de facteurs qui partout contribuent largement à l'extension des maladies vénériennes -, une campagne d'éclaircissement en grand style et surtout une très vaste organisation offrant à chaque vénérien un traitement gratuit et approprié. D'après lo $47^{\mathrm{me}}$ rapport du Local Government Board, les lois qui se rapportent à cette organasation et prévoient l'installation de centres pour le diagnostic et le traitement des maladies vénériennes, furent en I 9 I 8 élaborées sur un pied tel qu'environ 30,000,000 d'habitants pouvaient au besoin avoir recours à cette institution. On n'y a pas cncore introduit de véritables mesures de contrainte, sur le modèle des ordonnances suédoises ; mais je suis persuadé que leur création n'est qu'une question de temps, car là comme ailleurs ressort le fait que, bien qu'existantes, les institutions sont négligées par une grande partie des malades.

Je passe sous silence les dispositions prises dans ce même but par d'autres pays tels que la France, le Canada, l'Australie, l'Italie, et je termine cet exposé par un court aperçu de ce qui se faît dans les Etats-Unis d'Amérique. Comme on pouvait s'y attendre les mesures prises dans ce pays portent l'empreinte du grandiose. Elles découlent en partie d'institutions et de sociétés privées. A ce point de vue, il faut ici mentionner tout spécialement l'activité des femmes qui, préoccupées à juste titre des dangers que font courir aux générations futures les parents malades, ont décidé, lors d'un congrès tenu en septembre rgrg, d'entreprendre une grande campagne contre toute immoralité qui contribue à l'extension des maladies vénériennes.

Ainsi qu'il ressort du rapport officiel du service de santé (Public Health Reports), l'Etat lui-même, sans attendre l'initiative privée, a entrepris dans toute l'étendue du pays une campagne d'éducation et d'instruction. Elle consiste dans la distribution d'innombrables feuilles volantes, dans des conférences, dans des représentations cinématographiques, etc. La plupart des Etats ont ordonné des mesures exigeant de la part des médecins la dénonciation des cas de maladies véné- 


\section{La guerre et les maladies vénériennes.}

riennes observés par eux et fixant l'organisation du contrôle et de la lutte contre ces maladies.

J'attache une grande importance à la constatation officielle que cette façon d'agir n'a entraîné aucun des inconvénients que les adversaires de ce système lui ont si souvent reprochés "Infected persons have not refused treatment because report of the case by number or name was required. No report has reached the Public Health Service of injustice suffered by any infected person as a result of the case being reported etc. "Les malades au contraire trouvent tout intérêt à se faire traiter jusqu'à guérison puisqu'ils sont sûrs d'éviter ainsi toute perquisition ultérieure. Dans beaucoup d'Etats le charlatanisme est interdit et le traitement gratuit. Dans d'autres, par contre, la situation est encore loin d'être aussi avancée.

Je veux clore ici cet aperçu sur les principales mesures prises dans ce domaine et terminer par quelques considérations générales :

Mes études m'amènent à cette conclusion que la guerre mondiale et tout ce qui en découle a augmenté les maladies vénériennes dans une proportion jusqu'ici inouie. Il en est résulté que, sous la menace des dangers que cet état de choses a fait naître, les autorités militaires d'abord, puis civiles, des différentes nations ont tenté des efforts considérables pour opposer un rempart à l'extension du fléau.

Mais par là une petite partie seulement du travail qui doit être exécuté a été accomplie. La lutte sur les champs de bataille s'est apaisée, et l'humanité s'apprête à reconstruire petit à petit les innombrables œuvres matérielles et morales que la guerre a mises à néant. Le but de tous nos efforts doit être non pas seulement de revenir à la situation d'avant la guerre, mais encore d'apporter aux individus et aux peuples une existence meilleure et plus heureuse. Tout particulièrement doivent nous tenir a cœur l'hygiène du peuple et l'amélioration de la race, dont on ne saurait priser assez haut l'importance pour l'avenir de l'humanité civilisée. Au premier plan de cette amélioration s'impose la lutte contre les mala-

$$
-273-
$$




\section{La guerre et les maladies vénériennes.}

dies vénériennes, dont l'action néfaste sur la race se fait sentir plus que n'importe quelle autre défectuosité d'ordre physique. Car l'avenir a besoin d'hommes sains et robustes et non pas d'individus portant en eux le germe de maladies graves et progressives, néfastes encore pour les générations suivantes.

Il ne faut se faire aucune illusion, la lutte contre les maladies vénériennes est une dure entreprise, d'autant plus pénible qu'à sa réussite viennent s'opposer, à côté des obstacles physiques inévitables, toute sorte de préjugés d'ordre éthique et social, tenaces et depuis longtemps enracinés.

Il ne suffit pas de diminuer la contagion par mille moyens préservatifs, mais bien plutôt de déraciner et de détruire des mœurs et des opinions sexuelles transmises depuis des siècles. Cette lutte doit réussir et elle réussira. Car les maladies vénériennes ne sont pas une indestructible nécessité de la nature, mais un mal certainement évitable. Et de même que chaque individu parvient, lorsqu'il le veut fermement, à éviter la blennorrhagie et la syphilis, aussi bien cette possibilité existet-elle pour la collectivité.

Mais si ce but veut être atteint, il faut vraiment y consacrer des efforts bien plus considérables qu'avant la guerre. Ce n'est plus assez de quelques groupes restreints de médecins et de philanthropes; il faut remuer et mettre en mouvement les masses. Les femmes surtout, qui jusqu'à présent, au détriment du résultat, se sont tenues à l'écart, doivent entrer dans la lutte. Elles doivent comprendre qu'à la solution de ce problème est attaché leur bien le plus sacré, tant physique que moral. L'égalité des sexes dans ce domaine, au lieu du favoritisme accordé jusqu'à aujourd'hui au sexe mâle, représente déjà un énorme progrès. D'autant plus qu'il est bien plus facile de reconnaître dès le début et de soigner à fond une maladie vénérienne chez l'homme que chez la femme.

L'instruction des masses, la propagande sur une vaste échelle et le prosélytisme des sociétés privées sont des conditions primordiales et indispensables. A elles seules cependant, elles ne sauraient suffir. Sans l'intervention énergique de l'Etat 


\section{La guerre et les maladies vénériennes.}

le problème n'est pas soluble. A tous ceux qui craignent encore cette intervention $j$ 'adresse cette sentence extraite d'un rapport du service de santé des Etats-Unis : "Protection of the individual is subordinate to the preservation of the public health 》.

$\mathrm{Si}$ nous voulons voir surgir une amélioration dans un temps prochain, c'est à la seule condition que les particuliers ne reculent pas devant le sacrifice de désirs et de penchants égoïstes, dans l'intérêt du bien général. Ici, comme dans tous les grands mouvements et progrès de l'humanité, beaucoup d'optimisme et d'idéalisme est nécessaire, si l'on veut surmonter les obstacles et les contre-coups qui ne sont épargnés à aucune grande lutte. Qui combat pour le droit et $\mathrm{y}$ persévère sera toujours vainqueur.

Le problème de la lutte contre les maladies vénériennes n'est pas strictement national; il est de tous points international, et sa solution doit être cherchée sur le terrain international. Qui, mieux que la Croix-Rouge, pourrait mener ce combat? 